\title{
A Influência da Fé, Espiritualidade e Reliogiosidade sobre o tratamento de paciente com câncer - Relato de Caso.
}

\section{The Influence of Faith, Spirituality and Religiosity on the treatment of cancer patients - Case Report.}

\begin{abstract}
RESUMO
O diagnóstico de uma neoplasia pode determinar indesejadas e imprevistas repercussões na vida do paciente, afetando as suas dimensões físicas, psicológicas, social e econômica. Todo o processo do tratamento e a perspectiva de viver com uma enfermidade com paradigma de fatalidade podem desencadear reações emocionais negativas sistemicamente como: perda de apetite, insônia, alteração de humor... Ainda repercutir no aspecto psicossocial do mesmo como: medo do abandono (pela família e/ou amigos), temor de recidiva e da morte, e em muitos casos sobre a própria imagem como indivíduo. Este artigo demonstra a importância e o sentido que a fé, a religiosidade e a espiritualidade podem desempenhar sobre o diagnóstico e tratamento da doença de forma positiva ou negativa tornando assim, a fé aa religiosidade e a espiritualidade um fio condutor que integra a vivencia destes pacientes.

Palavras chave: carcinoma, religiosidade, oncologia.
\end{abstract}

\section{ABSTRACT}

The diagnosis of a neoplasm can determine unwanted and unforeseen repercussions in the patient's life, affecting its physical, psychological, social and economic dimensions. The entire treatment process and the prospect of living with a disease with a fatality paradigm can trigger negative emotional reactions systemically, such as: loss of appetite, insomnia, mood swings... It also affects the psychosocial aspect of it as: fear of abandonment ( by family and/or friends), fear of recurrence and death, and in many cases about one's own image as an individual. This article demonstrates the importance and meaning that faith, religiosity and spirituality can play on the diagnosis and treatment of the disease in a positive or negative way, thus making faith in religiosity and spirituality a common thread that integrates the lives of these patients .

Keywords: carcinoma, religiosity, oncology.

\section{Poliana Rodrigues Moraes ${ }^{1}$ \\ Pamela Aparecida Diniz ${ }^{2}$}

${ }^{1}$ Graduada em Odontologia e Pós-Graduada em Saúde Pública com Ênfase em Saúde da Família.

${ }^{2}$ Graduada em Odontologia, Mestrado em Biopatologia Bucal e Doutorado em Biopatologia Bucal.

Poliana Rodrigues Moraes - Rua João Basílio 643 - Centro, Pouso Alegre - MG, 37550-000 (35) 9-8829-3784 pollyrmoraes@gmail.com 


\section{INTRODUÇÃO}

Até 2025 um dentre os cinco principais problemas de saúde, segundo a Organização Mundial da Saúde (OMS), exceto região da África, será o câncer; passando assim a considera-lo como um grave problema de saúde pública (Zare A. Et al, 2019).

A neoplasia de cabeça e pescoço é um termo amplo que representa as neoplasias malignas que atingem as estruturas nesta região como cavidade oral, laringe, faringe e seios paranasais (Chen X. Et al,2017).

O carcinoma espinocelular (CECCP) é um dos diferentes tipos de câncer que se desenvolvem em pele. Sua origem esta associada em cavidade bucal ao consumo excessivo de drogas, alcool e fumo. Em pele costuma atingir áreas do corpo expostas ao sol, como rosto, orelhas, pescoço, lábios e dorso das mãos. Esta tem uma alta taxa de mortalidade associada com a alta prevalência em países de baixo nível socioeconômico. O CECCP é a sexta maior causa de morte por câncer no Brasil; sua incidencia é maior em homens que em mulheres entre a quarta e quinta décadas de vida (Chen X. Et al,2017).

Sabendo disto, o câncer pode significar ao paciente mais do que uma dor física. Ele interfere em seu modo de viver, na família, no trabalho, na renda e sua imagem corporal.; mudanças estas que podem ser temporárias ou permanentes. A ressignificação, a religiosidade e a espiritualidade podem atuar como um benefício ou não para o paciente (Zare A. Et al, 2019).

Todo ser humano é um individuo único, e sua multidimensionalidade demonstra a composição de fatores de inter-relações e interdependências, nos aspectos social, biológico, psicológico e espiritual. Desta forma qualquer um desses fatores ao sofrer algum prejuízo resultara em um ser humano com a saúde afetada (Ferreira, R. Et al, 2007).

As doenças precisam ser analisada sob seus diversos prismas. A perspectivas física refletido como injurias físicas como tumores e fraturas, a exemplo. A perspectiva psicologica onde cabe seu estado interno como suas emoções e pensamentos. A perspectiva cultural demonstrado pela visão comum do mundo a saber os valores religiosos, a relação familiar e destes valores, quais são ameaçados pela doença. A perspectiva social que envolve os as aspectos economicos e o relacionamento junto a comunidade (Matos TDS. Et al, 2017).

Quando da era moderna houve uma busca ininterrupta pela ciencia por inovações para sua expansão. Em paralelo a religiosidade surge em busca de preencher a incompletude humana, atendendo a necessidade do individuo de se desvincular momentaneamente a uma realidade dura, por vezes fria e materialista. Desta forma sua fé se faz presente ao adorar um Deus específico ou ao poder da natureza, à realização de caridade, entre outros (Sales CA. Et al, 2004).

Neste ponto torna-se importante a compreensão de que saúde e doença sofrem influências das diversas faces da perspectiva humana e cada face pede algo diferente para o individuo sob o ponto de vista dos valores individuais, concepções científicas, religiosas e filosóficas (Ferreira, R. Et al, 2007).

Quando enfatiza-se a religiosidade, embora seja sabido que muitas comunidades religiosas capacitem seus lideres, sabe-se também que muito do conhecimento do religioso advem de um saber nato e que produz conhecimento a partir de vivências e se expressa a visão de mundo (Zare A. Et al, 2019). 
Neste contexto destaca-se indagações sobre a compreensão de saúde e de doença, a influência da fé do enfermo sobre a cura de sua doença e principalmente sobre a adesão ao tratamento e continuidade do mesmo. Destaca-se ainda os meios aos quais o líder dispoem para atender as situações de doença das pessoas que o procuram, ou não (Matos

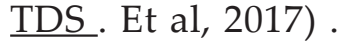

\section{METODOLOGIA}

As informações contidas neste trabalho foram obtidas através de revisão de literatura, revisão do prontuário, entrevista com o paciente, registro fotográfico dos métodos diagnósticos e terapêuticos ao qual o paciente foi submetido.

\section{RELATO DE CASO}

Paciente sexo feminino, leucoderma, 51 anos, casada, ensino fundamental incompleto e trabalhadora da limpeza urbana de empresa pública; apresentou nódulo circunscrito, palpável e sem ponto de flutuação, tamanho aproximado $04 \mathrm{~cm}$, em região cervical a direita do qual a referida não se queixava de dor.

Ao ser sugestionado a iniciar investigação para posterior tratamento a mesma recusou e evadiu da unidade de atendimento. Durante o período de evolução da doença passou a utilizar xales, lenços e blusas com gola alta para esconder a lesão (sic). Recusava qualquer estimulo para procura de assistência médica devido ao seu alto fervor religioso (sic), inclusive estimulo este vindo dos representantes de sua própria igreja.

Com estrutura familiar bastante deficiente; paciente possui dois filhos (26 e 29 anos) com importante comprometimento cognitivo sendo o mais velho o de maior grau. Ambos completaram o ensino fundamental e foram retirados da escola pela mãe (sic) não iniciando

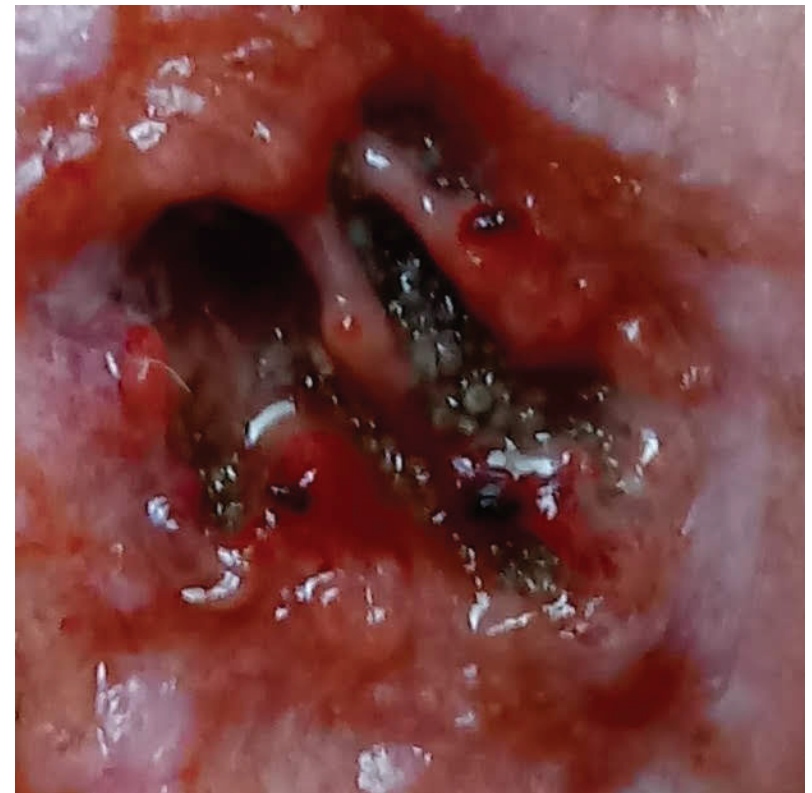

Figura 1 o ensino médio. Ainda, não era incomum que seus filhos a ajudassem na varrição (local de trabalho desta mãe), desde párvulos.

Em 90 dias após o primeiro contato, a paciente retornou à unidade por insistência de colega de trabalho. Na presente data foi observado que a lesão se apresentava ulcerada sem delimitação, tomada por miíase, odor fétido e sistemicamente pode-se observar mal estado geral e febre.

Procedeu-se internação hospitalar onde teve inicio limpeza da lesão com remoção da miíase (Figura 1), antibióticoterapia e investigação de origem da lesão com biopsia incisional em ambulatório, tomografia de pescoço (Figura 2) e o fibronaso (Figura 3); o primeiro por sua vez trouxe como resultado a inconclusividade (figura 4). 


\section{TC DE PESCOÇO COM CONTRASTE 05/11/18}

FORMAÇÃO EXPANSIVA COM DENSIDADE E REALCE PELO CONTRASTE HETEROGENEOS, COM FOCOS
CALCICOS ESPARSOS, LOCALIZADO

EM REGIĀO CERVICAL POSTEROLATERAL DIREITA, POSTERIOR AO MÚSCULO

ESTERNOCLEIDOMASTOIDEO, MEDINDO $13,5 \times 13,5 \times 11,3$

CM (LL X AP X T), ASSOCIADO A ULCERAÇÃO SUPERFICIAL COM FOCOS GASOSOS DE PERMEIO,
ACARRETANDO DESVIO DA LINHA

MÉDIA CONTRALATERALMENTE, SENDO INDISSOCIÁVEL DO LOBO DIREITO DA TIREOIDE E DA
CARTILAGEM CRICÓIDE DIREITA.

Figura 2

\section{FIBRONASO 07/11/18}

PROCESSO EXPANSIVO SUBMUCOSO AO NIVEL DA OROFARINGE A DIREITA

Figura 3

ANATOMOPATOLOOGICO (BIOPPIA L.ESÄO CERVICAL) 09/11/18

O EXAME MICROSCÓPICO EVIDENCIA CORTES HISTOL.OGICOS DE MATERIAL CONSTITUIDO POR

NECROSE, 'TECIDO DE GRANULAÇAO E AREAS DE SUPURAÇAO, AO LADO DE FRAGMENTO DE PELE

Figura 4

Entretanto apenas 10 dias após sua internação a paciente assinou Termo de Responsabilidade e evadiu do ambiente hospitalar verbalizando sua preferência pelo tratamento espiritual.

Decorridas 48 horas a mesma apresentou declínio de seu estado geral e sangramento abundante advindo da lesão e novamente deu entrada em ambiente hospitalar.

Em sua segunda internação pode-se realizar nova biopsia incisional (Figura 5) e coletar material para realização do imunohistoquimico que teria o resultado liberado aproximadamente 60 dias apos a coleta.

ANATOMOPATOLOGICO (BIOPSIA LESAOO CERVICAL) 13/12/18

NEOPLASIA MAL IONA POUCO DIFERENCIADA NA MOSTRA DA BIOPSIA E APRESENTANDO AS

SEGUINTES CARACTERISTICAS:

ORAU DE ANAPLASIA NUCLEAR: ALTO GRAU.

CEIUIARIDADE: ADUNDANTE.

INDICE MITÓTICO: 31 MITOSES EM 10/ CGA.

NECROSE TUMORAL: PRESENTE (MODERADA)

INVASAO VASCULAR ANOIOL.INFATICA: NAO DETECTADA.

INVASAO PERINEURAL: NAO DETECTADA.

Figura 5

Exames evidenciaram neoplasia maligna altamente invasiva e com alto grau de diferenciação celular com expansão submucosa em direção a orofaringe à direita. Nota-se ainda comprometimento renal, anemia por deficiência de ferro e plaquetopenia.

$\mathrm{Na}$ oportunidade uma sonda nasoenteral foi instalada para que a dieta pudesse ser ofertada a paciente. 
Foram realizadas 10 sessões de radioterapia hemostática a fim de reduzir o sangramento oriundo da lesão (figura 6).

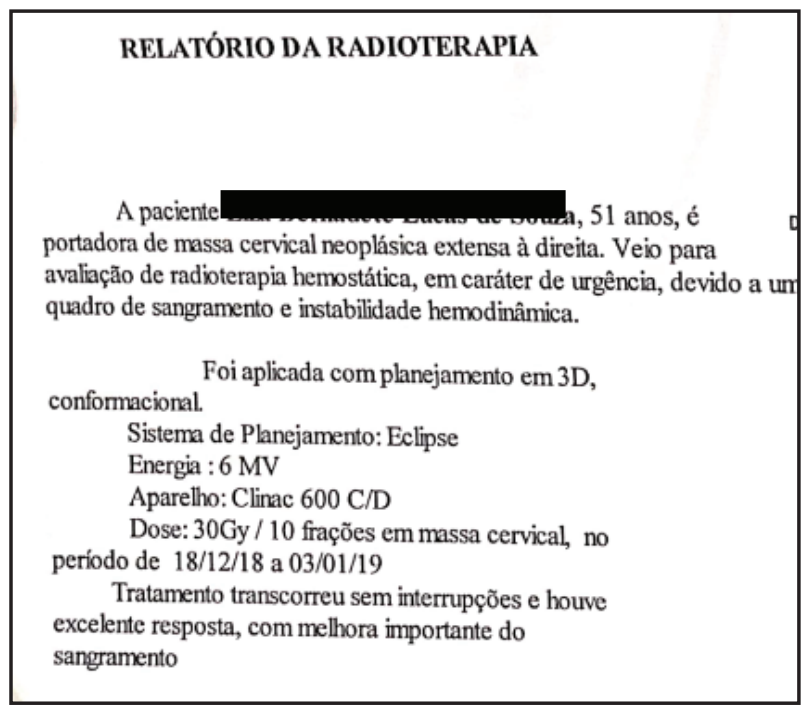

Figura 6

Decorridos 30 dias de sua segunda internação e já em alta hospitalar o imunohistoquímico da paciente evidenciou carcinoma de células escamosas, pouco diferenciadas (figura 7).

Diaquóstico:

BIÓPSIA DE LESÃO CERVICAL: CARCINOMA DE CÉLULAS ESCAMOSAS VER COMENTÁRIO.

\section{Comentário:}

Os cortes mostram fragmentos de pele exibindo neoplasia maligna de padrão epitelioide eosinofílico, com núcleos volumosos e nucléolos proeminentes (foto 1). O estudo imuno positividade para o anticorpo p63 (foto 2) e para citoceratinas (foto 3). Estes achados co CARCINOMA DE CÉLULAS ESCAMOSAS POUCO DIFERENCIADO. O diagnóstico definiti consideração os achados clínicos e radiológicos.

Estudo imuno-histoquímico: Após desparafinação e tratamento dos tecidos com soluções próprias para recuperaç incubados com painel de anticorpos monoclonais elou policlonais. A seguir utilizou-se o sistema de deteç̧äo bas negativos foram utilizados para atestar a fidelidade das reações. Resultados individuais para os marcadores estud resultados especificados dizem respeito às células de interesse no contexto de cada caso.

\begin{tabular}{lll}
\hline Anticorpos & Clone & Resultad \\
\hline - Citoceratinas de 40, 48, 50 e $50,6 \mathrm{kDa}$ & AE1/AE3 & Positivo \\
- Melan A (MART-1), antígeno do melanoma reconhecido por células T & A103 & Negativo \\
- Proteína S-100 & Policlonal & Negativo \\
- Proteína p63 (epitélios escamoso/transicional; células mioepiteliais) & EP174 & Positivo \\
Bibliografia: & & \\
1. AK El-Naggar, JKC Chan, JR Grandis, T Takata, PJ Slootweg (Eds): World Health Organization Classification of Head and Neck Tu \\
2. Bruce M. Wenig, MD. Atlas of Head and Neck Pathology. Saunders; 3rd edition, 2015. \\
\end{tabular}

Figura 7 
Como tratamento adotado iniciou-se os cuidados paliativos, realizados pela ESF, com curativos diários e fármacos analgésicos em primeira escolha: Dipirona $1 \mathrm{~g}$, em segunda escolha Tramadol 100mg e em última escolha Morfina 10mg; devido ao mal estado geral da paciente e diminuição da função renal a quimioterapia fora descartada. A cirurgia de remoção da tumoração (Figura 8,9,10) foi contra-indicada devido a sua alta vascularização.

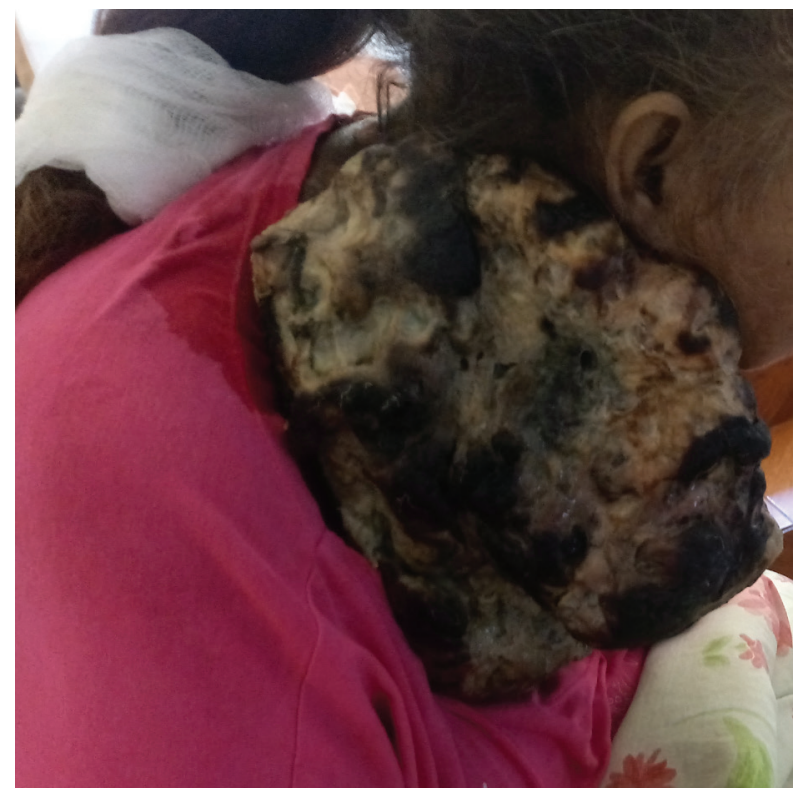

Figura 8

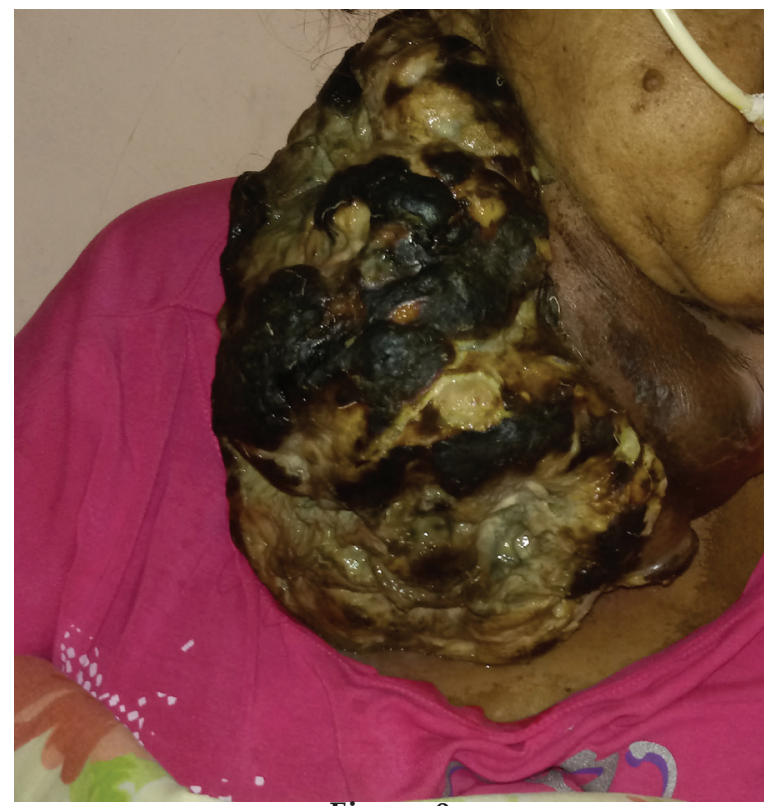

Figura 9

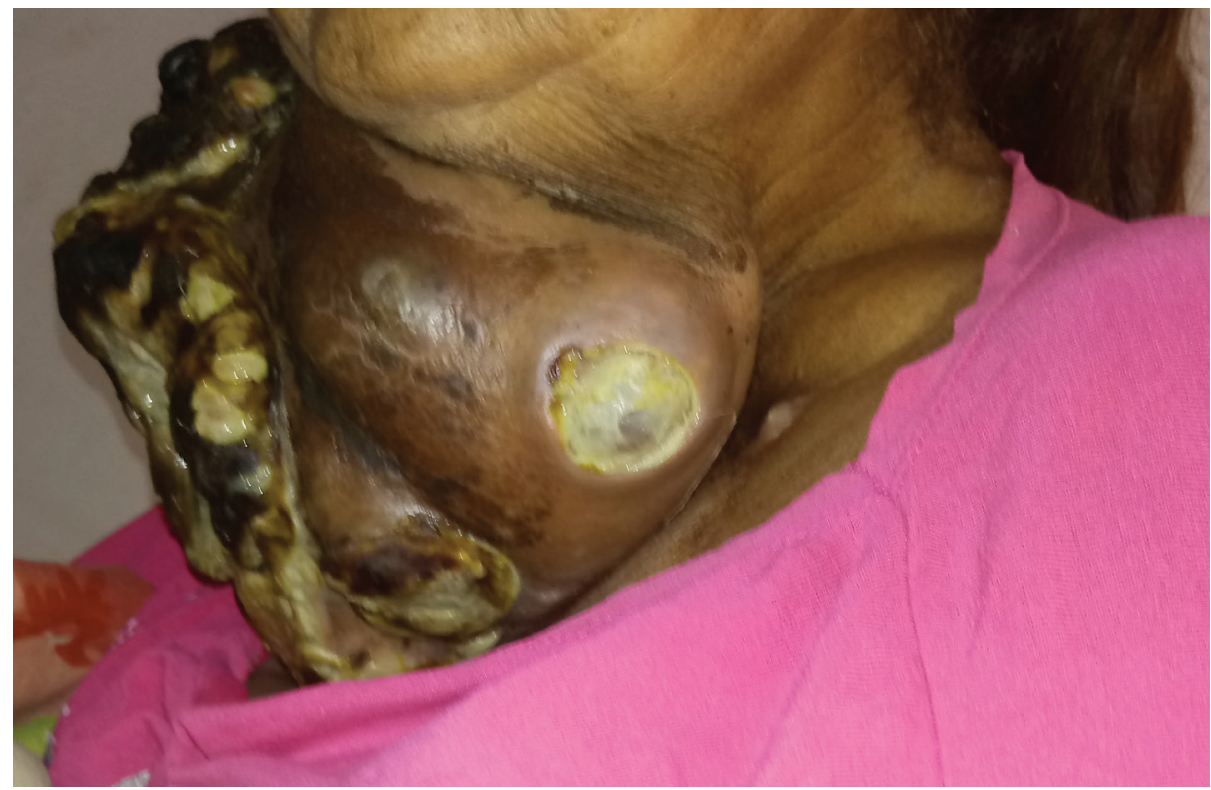

Figura 10 
Nota-se extrema resistência da paciente em permitir a entrada da equipe de saúde da família em sua residência para a execução dos procedimentos básicos solicitados pela oncologia quando de sua alta hospitalar.

A paciente sempre fora resistente á estímulos externos. A primeira vez que a equipe de saúde da família pode, com permissão, entrar em sua residência ocorreu quando a mesma evadiu o hospital assinando o termo de responsabilidade.

Quando da fala, a paciente é bastante enfática em afirmar que a cura de sua patologia virá de sua fé.

Quarenta e cinco dias após alta hospitalar a paciente veio a óbito em ambiente domiciliar.

\section{DISCUSS ÃO}

Na literatura o apoio em Deus também fora descrito para enfrentamento de situações limitantes como uma necessidade (Fischer I. Et al, 2019).

Para Paiva BS. Et al, 2015 neoplasia é uma doença estigmatizada, consigo traz a ideia de morte e sofrimento onde o processo de cura é considerado pelos enfermos como um milagre e não como algo passível de ocorrer, mesmo com os avanços da medicina e isso acaba criando barreiras à prevenção.

O desenvolvimento de uma neoplasia leva o ser humano a deparar-se com seus valores e com outras questões como, a mais pertubadora, existência e a proximidade da morte. Assim a religião e a espiritualidade compreendem o esforço de ressignificar essa nova etapa da vida que fora apresentada para o paciente, onde ocorre a busca pela compreenção da própria doença, do sofrimento, da morte e da existência (Vallurupalli M. Et al, 2012).

Para Sales CA. Et al, 2004, a luta de mulheres contra as neoplasias malignas, fora sustentada baseado na esperança e confiança em um ser supremo. Ainda para Vallurupalli M. Et al,2012 ter a segurança em depositar situações que desorganizam a vida ao sagrado, motiva o enfermo ao enfrentamento onde os percausos tornam-se previstos, o acaso e não existe fatalidade.

A relação entre a espiritualidade e o câncer foi sintetizada por Guerrero P; 2011 como: O câncer como o que amedronta e a espiritualidade como aquela que renova.

A abordagem de patologias debilitantes são em sua maioria de dificil manejo pelos pacientes e/ou cuidadores do mesmo. Estes passam por diferentes fases desde a descoberta até o tratamento de sua doença. Num primeiro momento, ocorre um estado de choque, em seguida a realidade do fato, passando pelo estagio de não saber o que pensar, e finalmente com a crença em um ser supremo passam a planejar o futuro com esperança (Jafari N. Et al, 2013).

Dessa forma, é necessário considerar a dimensão espiritual do paciente para abordar a esperança e o enfrentamento da doença no planejamento da assistência, e, para isso, é fundamental conhecer a visão de mundo e a cultura à qual ele pertence (Fischer L. Et al, 2019)..

Para FARIA (2005), ao paciente cabe reconhecer seu direito em buscar alternativas que o encaminhem ao bem estar, entretanto eles não devem interferir no tratamento médico. Reconhecer a importância do tratamento medicamentoso mesmo recorrendo à religiosidade/ 
espiritualidade. demonstra positiva relação ao processo saúde-doença/fé. A ultima pode ser positiva ao paciente quando o flexibiliza a adesão do tratamento e não o contrario.

\section{CONCLUSÃO}

A espiritualidade pode ser uma forma bastante importante para que o paciente enfrente uma doença como o câncer, já que ao próprio despendirá a atribuição de significado ao seu processo saúde-doença, em busca da sobrevivência, acalento e esperança a fim de minimizar o seu sofrimento ou evoluir durante o tratamento com maior acreditação na cura, considerando a sua percepção individual.

A espiritualidade trata-se de de um tema subjetivo e amplo, e tal imprevisibilidade encaminhará cada paciente a depender de suas atitudes perante o todo, resultados diferentes.

Quando o amparo religioso se torna negativo o paciente com câncer utiliza-se de sua fé para justificar comportamentos negligentes em saúde, ou substituir tratamentos médicos. Entretanto em sua maioria a fé quando aliado às recomendações clínicas, ajuda no encorajamento ao enfrentamento da doença e contribui beneficamente nas funções orgânicas por meio de efeitos psiconeuroimunológicos, modulação da frequência respiratória e cardíaca, além de atuar na diminuição do estresse.

\section{REFERÊNCIAS}

Zare A , Bahia NJ , Eidy F , Adib N , Sedighe F . The relationship between spiritual well-being, mental health, and quality of life in cancer patients receiving chemotherapy. J Family Med Prim Care. 2019 May;8(5):1701-1705. doi: 10.4103/jfmpc.jfmpc_131_19

Chen X , Yuan Z, Lu M , Zhang Y, Jin L. Et al. Poor oral health is associated with an increased risk of esophageal squamous cell carcinoma - a population-based case-control study in China. Int J Cancer. 2017 Feb 1;140(3):626-635. doi: 10.1002/ijc.30484. Epub 2016 Nov 7.

Ferreira, R. E. R., \& Fornazari, S. A. (2007). A influência da fé na qualidade de vida em pacientes oncológicos. Relatório Final de Trabalho de Conclusão de Curso, Universidade Paulista, Assis.

Matos TDS , Meneguin S , Ferreira MLDS , Miot HA . Quality of life and religious-spiritual coping in palliative cancer care patients. Rev Lat Am Enfermagem. 2017 Jul 10;25:e2910. doi: 10.1590/1518-8345.1857.2910.

Fischer J , Stope MB , Gümbel D , Hakenberg O , Burchardt M , et al (2019).Influence of culture and religion on the treatment of cancer patients. Urologe A. 2019 Oct; 58 (10): 1179-1184. doi: 10.1007 / s00120-019-1003-5.

Paiva BS, Carvalho AL, Lucchetti G, Barroso EM, Paiva CE. “Oh, yeah, I'm getting closer to god": spirituality and religiousness of family caregivers of cancer patients undergoing palliative care. Support Care Cancer. 2015 Aug;23(8):2383-9. doi: 10.1007/s00520-015-2604-1. Epub 2015 Jan 16.

Vallurupalli M , Lauderdale K, Balboni MJ , Phelps AC , Bloco SD. et all The role of spirituality and religious coping in the quality of life of patients with advanced cancer receiving palliative radiation therapy. J Support Oncol. 2012 Mar-Apr;10(2):81-7. doi: 10.1016/j.suponc.2011.09.003. Epub 2011 Nov 16.

Sales CA, Molina MAS. O significado do câncer no cotidiano de mulheres em tratamento quimioterápico. Rev Bras Enferm 2004; 57(6): 720-3.

Guerrero, P. Relação entre espiritualidade e câncer: perspectiva do paciente. Rev Bras Enferm, Brasília 2011 jan-fev; 64(1): 53-9.

Jafari N, Farajzadegan Z, Zamani A, Bahrami F, Emami H, et al. Spiritual well-being and quality of life in Iranian women with breast cancer undergoing radiation therapy.Support Care Cancer. 2013 May;21(5):1219-25. doi: 10.1007/s00520-012-1650-1. Epub 2012 Nov 10.

Faria JB, Seidl EMF. Religiosidade e enfrentamento em contextos de saúde e doença: revisão da literatura. Psicol Reflex Crit 2005; 18(3): 381-9. 